\title{
Quantized acoustoelectric current in a finite-length ballistic quantum channel: The noise spectrum
}

\author{
Y. M. Galperin ${ }^{(a)}$, O. Entin-Wohlman ${ }^{(b)}$ and Y. Levinson ${ }^{(c)}$ \\ (a) Department of Physics, University of Oslo, Box 1048 Blindern, N-0316 Oslo, Norway \\ and Solid State Division, A. F. Ioffe Physico-Technical Institute, 194021 St. Petersburg, Russia \\ (b) School of Physics and Astronomy, Raymond and Beverly Sackler Faculty of Exact Sciences, \\ Tel-Aviv University, Tel-Aviv 69978, Israel \\ ${ }^{(c)}$ Department of Condensed Matter Physics, The Weizmann Institute of Science, Rehovot 76100, Israel
}

(November 1, 2018)

\begin{abstract}
Fluctuations in the acoustoelectric current, induced by a surface acoustic wave propagating along a ballistic quantum channel, are considered. We focus on the large wave-amplitude case, in which it has been experimentally found that the current is quantized, and analyze the noise spectrum. A phenomenological description of the process, in terms of a random pulse sequence, is proposed. The important ingredients of this description are the probabilities, $p_{n}$, for a surface acoustic wave well to capture $n$ electrons. It is found that from the noise characteristics one can obtain these probabilities, and also estimate the typical length scales of the regions in which the electrons are trapped.
\end{abstract}

Acoustic methods appear to be extremely useful in probing physical properties of the two-dimensional electron gas (2DEG) [1]. Surface acoustic waves (SAW's) induce long-range electric fields, with the spatial and temporal periodicities of the wave. These fields, which penetrate the 2DEG without any galvanic leads, facilitate a probeless diagnostics. In addition, the energy loss in such experiments takes place inside a very small volume which is well thermally coupled to the substrate. As a result, SAW's usually do not cause overheating of the electronic device.

Two effects are customarily being studied. The first is the attenuation of the SAW's due to electric currents induced in the 2DEG. This effect, which is linear in the acoustic amplitude, allows the determination of the linear response of the 2DEG to an ac perturbation, of frequency and wave vector of the acoustic wave. For example, studies of the acoustic attenuation and the velocity in the presence of an external magnetic field perpendicular to the 2DEG have been exploited to investigate the ground state of two-dimensional quantum Hall effect systems [2]. The second is the acoustoelectric effect, which a nonlinear response. In a quantum channel, this effect has been measured in Ref. [3]. The electric fields induced by SAW's at the 2DEG drag the electrons, and consequently produce a dc current in a closed circuit, or a dc voltage across an open circuit.

Recently it has been experimentally demonstrated [4] that under proper conditions the acoustoelectric current through a non-biased pinched-off channel in a 2DEG consists of a set of plateaus, when the gate voltage, or alternatively the SAW amplitude, is varied. Below a certain threshold of the SAW intensity the current is very small (the structure of the current in that regime has been discussed in Refs. [4, ,7,8]); at the threshold it "jumps" to a quantized value, ef, (where $e$ is the electron charge and $f$ is the SAW frequency), which it keeps up to a second threshold; then it "jumps" again to a second quantized value $2 e f$.

Quantization of the current carried by electrons trapped in a moving potential has been first addressed in Ref. [9] where it has been shown that the current induced by a slowly moving periodic potential can be quantized in units of $e L / v$ where $L$ is the period of the potential profile and $v$ is its velocity. Previous theoretical considerations have addressed the microscopic origins of the quantized current 10 12]. Here we also consider the relatively large SAW amplitude case, but propose a phenomenological description of the quantized current and its noise, in terms of a random pulse sequence. The picture we have in mind stems from the quantitative explanation outlined in Refs. 14 . 6 .

In the absence of the SAW, the quantum channel in the 2DEG is pinched-off, that is, the common Fermi level $\epsilon_{F}$ in the (non-biased) source and drain is below the bottom of the channel band, $\epsilon_{B}$, and so electrons from the terminals cannot penetrate the channel (see Fig. 1a). When a SAW is propagating along the channel, the bottom of the channel band is modulated by the moving piezoelectric potential profile, which consists of wells separated by barriers. Such a modulation does not take place in the terminals because the 2DEG strongly screens the effect of the wave. The potential profiles at different times are schematically depicted in Fig. 1b,c. For a strong enough SAW, the modulation amplitude $V_{\text {SAW }}$ exceeds the difference $\epsilon_{B}-\epsilon_{F}$. Then the bottoms of the wells that appear near the source during half of the period of the SAW, are located below the Fermi level (see Fig. 1b). Such a well may trap one or more electrons from the source into bound states $\epsilon_{0}$ in the well and then drag them along 
toward the drain. Upon the arrival of the well at the drain, the electron energies $\epsilon_{0}$ are below $\epsilon_{F}$ (see Fig. 1b). However, the electrons are adiabatically excited to $\epsilon_{F}$ because of the squeezing of the well as it disappears into the drain, (compare the levels in the right well in Fig. 1b and Fig. 1c) and the electrons are eventually absorbed by the drain.

Because of the strong Coulomb repulsion between two electrons on the same localized state, it is plausible that a not-too-deep well will capture only a single electron, a deeper well will accommodate two electrons, and so on. Hence, ignoring for the time-being any randomness or fluctuations in the process, the current induced by a moderate-intensity SAW can be viewed as a periodic sequence of non-overlapping pulses, whose period is the same as that of the SAW, $f^{-1}$, with each pulse, $i_{1}(t)$, carrying a single electron. On the average, such a sequence carries the current ef; as the intensity of the SAW's is increased and their wells become deeper, then pulses capable of carrying two electrons, $i_{2}(t)$, appear, leading to an average current $2 e f$, and so on. In this way, one obtains a qualitative picture of the phenomenon of the acoustoelectric current quantization [4] 6].

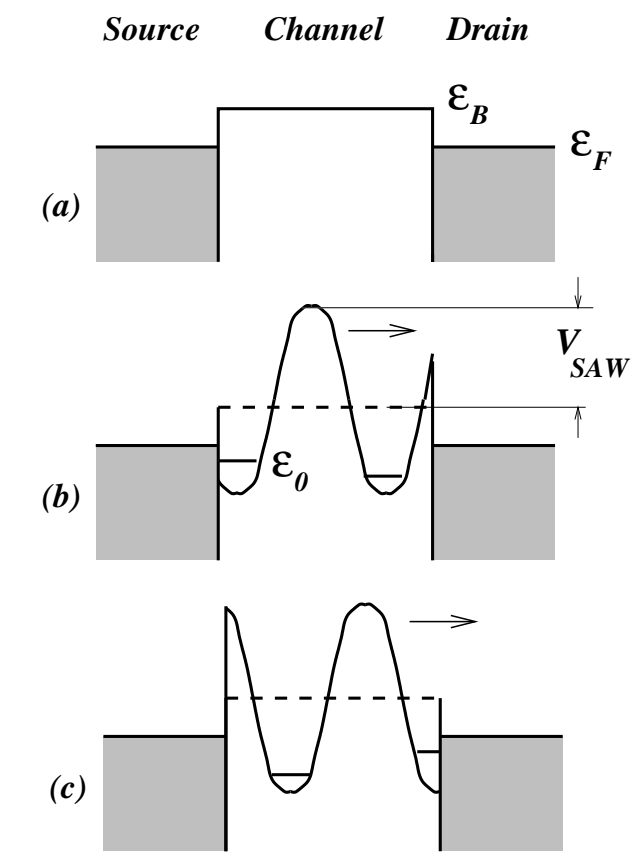

FIG. 1. Schematic profiles of the potential created by the SAW at different times.

However, naturally the acoustoelectric current is subject to fluctuations. Fluctuations will occur since some of the potential wells will not behave according to the scheme outlined above. For example, near and above the first threshold, there may be empty potential wells, which do not carry electrons; near the second threshold there will be wells carrying only one electron, and the current will be a random sequence of the pulses $i_{1}(t)$ and $i_{2}(t)$.
We hence propose a description in which the acoustoelectric current consists of a random sequence of pulses. Let $p_{n}$ be the probability that a potential well near the source captures $n$ electrons, and therefore contributes to the current the pulse $i_{n}(t)$, such that $\int_{-\infty}^{\infty} d t i_{n}(t)=n e$. We will show that the probabilities $p_{n}$, which depend on the temperature and on the intensity of the SAW's, can be extracted from the low-frequency fluctuations of the acoustoelectric current. These probabilities can be therefore directly probed by the experiment. Moreover, we will find that the fluctuation spectrum also provides information regarding the characteristic size, $a_{n}$, of the $n$-th electronic state confined in the well.

In our model, the current is thus a stationary random function of time,

$$
\mathrm{I}(t)=\sum_{\nu} \mathrm{F}_{\nu}\left(t-t_{\nu}\right)
$$

with $\mathrm{F}(t)$ being any of the pulses $i_{n}(t)$, with probability $p_{n}, \sum_{n} p_{n}=1$, and $t_{\nu}$ denotes the arrival time of the $\nu$ th pulse at the drain. The average time interval between the pulses, $\langle\tau\rangle$, is determined by the SAW period, i.e., $\langle\tau\rangle=f^{-1}$.

We next calculate the average current, and its fluctuation spectrum. To this end, it will be convenient to define the Fourier transform of a single pulse,

$$
\mathrm{F}_{\nu}(\omega)=\int_{-\infty}^{\infty} \frac{d t}{2 \pi} \mathrm{F}_{\nu}(t) e^{i \omega t}
$$

Consider first the average current. One has

$$
\langle\mathrm{I}(t)\rangle=\left\langle\int_{-\infty}^{\infty} d \omega e^{-i \omega t} \mathrm{~F}(\omega)\right\rangle\left\langle\sum_{\nu} e^{i \omega t_{\nu}}\right\rangle,
$$

as the pulse shapes and the arrival times are assumed to be statistically independent. The second average here is carried out over a time interval $T$ much longer than the duration of the pulses [13], yielding $\left\langle\exp \left(i \omega t_{\nu}\right)\right\rangle=$ $2 \pi \delta(\omega) / T$. The first average then gives

$$
N \sum_{n} p_{n} \int_{-\infty}^{\infty} d t i_{n}(t)=N e \sum_{n} p_{n} n,
$$

where $N$ is the total number of pulses, such that $\langle\tau\rangle=$ $T / N$. As a result, the average of the random stationary current is

$$
\langle\mathrm{I}(t)\rangle=\frac{e}{\langle\tau\rangle} \sum_{n} p_{n} n .
$$

As is mentioned above, the probabilities $p_{n}$ are functions of the SAW intensity. We hence conclude that below the first threshold of the measured current, the dominant probability is $p_{0}$; at the threshold $p_{1}$ becomes comparable to $p_{0}$, and above it, at the plateau, $p_{1}$ increases on the expense of $p_{0}$, and so on. 
The noise spectrum of the current, $\mathrm{S}(\omega)$, is defined as (see e. g. Ref. 14]),

$$
\mathrm{S}(\omega)=2 \int_{-\infty}^{\infty} d t e^{i \omega t}\langle\delta \mathrm{I}(t) \delta \mathrm{I}(0)\rangle,
$$

with $\delta \mathrm{I}(t)=\mathrm{I}(t)-\langle\mathrm{I}\rangle$. To calculate this quantity we follow Ref. [13]. One then finds [15]

$$
\begin{aligned}
\mathrm{S}(\omega) & =\frac{8 \pi^{2} N}{T}\left\langle|\mathrm{~F}(\omega)|^{2}\right\rangle \\
& +\frac{8 \pi^{2}}{T}|\langle\mathrm{~F}(\omega)\rangle|^{2}\left\langle\sum_{\nu^{\prime} \neq \nu} e^{i \omega\left(t_{\nu}-t_{\nu^{\prime}}\right)}\right\rangle .
\end{aligned}
$$

The last average here requires the distribution function of the arrival time differences (which has the average $\langle\tau\rangle$ ). Denoting it by $w(\tau)$, and introducing

$$
\phi(\omega) \equiv\left\langle e^{-i \omega \tau}\right\rangle=\int_{0}^{\infty} d \tau e^{-i \omega \tau} w(\tau)
$$

it is straightforward to find

$$
\left\langle\sum_{\nu^{\prime} \neq \nu} e^{i \omega\left(t_{\nu}-t_{\nu^{\prime}}\right)}\right\rangle=2 N \operatorname{Re} \frac{\phi(\omega)}{1-\phi(\omega)},
$$

in the $N \rightarrow \infty$ limit.

The result for the current fluctuation spectrum is conveniently presented in the form

$$
\mathrm{S}(\omega)=\mathrm{S}_{b}(\omega)+\mathrm{S}_{c}(\omega) .
$$

Here, $\mathrm{S}_{b}(\omega)$ is a smooth "background" noise, which is not affected by the distribution of intervals between pulses

$$
\begin{array}{r}
\mathrm{S}_{b}(\omega)=\frac{8 \pi^{2}}{\langle\tau\rangle}\left(\mathrm{K}(\omega)-|\mathrm{H}(\omega)|^{2}\right), \\
\mathrm{K}(\omega)=\left\langle|\mathrm{F}(\omega)|^{2}\right\rangle, \quad \mathrm{H}(\omega)=\langle\mathrm{F}(\omega)\rangle .
\end{array}
$$

In our model

$$
\mathrm{K}(\omega)=\sum_{n} p_{n}\left|i_{n}(\omega)\right|^{2}, \quad \mathrm{H}(\omega)=\sum_{n} p_{n} i_{n}(\omega) .
$$

The second term in Eq. (10) results from the fluctuations of the intervals among the arrival times of the pulses,

$$
\mathrm{S}_{c}(\omega)=\frac{8 \pi^{2}}{\langle\tau\rangle}|\mathrm{H}(\omega)|^{2} \mathrm{M}(\omega),
$$

where

$$
\mathrm{M}(\omega)=1+2 \operatorname{Re} \frac{\phi(\omega)}{1-\phi(\omega)} .
$$

There are various sources for the fluctuations of the intervals among the arrival times of the pulses, given by the distribution function $w(\tau)$, see Eq. (8). One is the instability in the SAW source, which is extremely small.
Another, even more important, is fluctuations originated from the instability of the gate voltages, which lead to fluctuations in the electron density and consequently in the sound velocity, due to the renormalization effect of the interaction between the SAW's and the 2DEG. In any event, it is expected that the distribution $w(\tau)$ is very narrow, such that the fluctuations, $\delta \tau=\tau-\langle\tau\rangle$, of those intervals are small, $\delta \tau \ll \tau$. In other words, $w(\tau)$ is strongly peaked around $\langle\tau\rangle=f^{-1}$. When the width of that peak is ignored, that is, $w(\tau)=\delta(\tau-\langle\tau\rangle)$, the function $\mathrm{M}(\omega)$ will vanish, unless $\omega$ coincides with the harmonics of the SAW frequency $\omega_{k}=2 \pi k f$, i.e.

$$
\mathrm{M}(\omega)=\frac{2 \pi}{\langle\tau\rangle} \sum_{k \neq 0} \delta\left(\omega-\omega_{k}\right) .
$$

As a result, $S_{c}(\omega)$ consists of a comb-like set of spikes. When the dispersion of the interval distribution is taken into account, these spikes are smeared. Their shapes can be found [13] by using for the function $\phi$, Eq. (8), the approximation

$$
\phi(\omega)=e^{-i \omega\langle\tau\rangle}\left[\left(1-\omega^{2}\left\langle(\delta \tau)^{2}\right\rangle / 2\right] .\right.
$$

A straightforward calculation then shows that the width of the $k$-th spike at its half-height is

$$
(\delta \omega)_{k} / \omega_{k}=2 \pi k\left\langle(\delta \tau)^{2}\right\rangle /\langle\tau\rangle^{2},
$$

and its height is given by

$$
\mathrm{M}\left(\omega_{k}\right)=4 / \omega_{k}^{2}\left\langle(\delta \tau)^{2}\right\rangle .
$$

In-between the spikes $\mathrm{M}(\omega)$ is small; for example, at the mid-point between successive spikes, where $\omega=$ $2 \pi(k+1 / 2) f$, its value is $2 \pi^{2}(k+1 / 2)^{2}\left\langle(\delta \tau)^{2}\right\rangle /\langle\tau\rangle^{2}$. Finally, at small frequencies one may write

$$
\phi(\omega)=1-i \omega\langle\tau\rangle-\omega^{2}\left(\left\langle(\delta \tau)^{2}\right\rangle+\langle\tau\rangle^{2}\right) / 2,
$$

to obtain $\mathrm{M}(0)=\left\langle(\delta \tau)^{2}\right\rangle /\langle\tau\rangle^{2}$.

Let us now discuss these results, in view of the experimental findings. Near the first threshold of the acoustoelectric current, it is plausible to assume that the potential wells capture either one electron, or none at all. One then finds, using $p_{0}+p_{1}=1$ in Eqs. (11) and (12), that the noise background is given by

$$
\mathrm{S}_{b 1}(\omega)=8 \pi^{2} f p_{0} p_{1}\left|i_{1}(\omega)\right|^{2} .
$$

Similarly, near the second threshold, the wells capture either one or two electrons, $p_{1}+p_{2}=1$, and one finds

$$
\mathrm{S}_{b 2}(\omega)=8 \pi^{2} f p_{1} p_{2}\left|i_{1}(\omega)-i_{2}(\omega)\right|^{2} .
$$

In both situations the fluctuations are strongest at the corresponding thresholds, where both relevant probabilities are close to $1 / 2$. This is reminiscent of the shot noise in point-contacts, which is proportional to $\mathrm{T}(1-\mathrm{T})$ 
(where $\mathrm{T}$ is the transmission through the point-contact) and has its maximum at the conductance steps [16].

The spectral width, $\Delta \omega$, of the noise is determined by the spectral width of the pulses $i_{n}(t)$. The duration of these pulses is $a_{n} / s$, where $s$ is the SAW velocity and $a_{n}$ is the localization length of $n$ electrons in the SAW well. This length depends on microscopic details, but is expected to be some fraction, $\eta<1$, of the SAW half-wavelength $s / 2 f$. Hence $\Delta \omega \simeq 2 f / \eta$ (presumably, $\eta$ is small). At frequencies well below the width $\Delta \omega$, $\omega \ll \Delta \omega$, one may then write $i_{n}(\omega) \simeq n e / 2 \pi$, to find from Eqs. (19) and (20)

$$
\begin{aligned}
& \mathrm{S}_{b 1}(0)=2 e^{2} f p_{0} p_{1} \equiv 2 e\langle\mathrm{I}\rangle \mathcal{F}_{1}, \quad \mathcal{F}_{1}=p_{0}, \\
& \mathrm{~S}_{b 2}(0)=2 e^{2} f p_{1} p_{2} \equiv 2 e\langle I\rangle \mathcal{F}_{2}, \quad \mathcal{F}_{2}=\frac{p_{1} p_{2}}{p_{1}+2 p_{2}} .
\end{aligned}
$$

Here, $\mathcal{F}_{i}$ are the Fano factors, which indicate the suppression of the noise compared to Poissonian noise. At the thresholds the suppression is moderate, $\mathcal{F}_{1}=1 / 2$ and $\mathcal{F}_{2}=1 / 6$, while far from them it is strong: $\mathcal{F}_{1}=p_{0} \ll 1$ and $\mathcal{F}_{2}=p_{2} \ll 1$ below the threshold and $\mathcal{F}_{2}=p_{1} / 2 \ll 1$ above.

As is mentioned above, the comb-like part of the spectrum, $\mathrm{S}_{c}$, consists of spikes located at $\omega_{k}=2 \pi k f$, with an envelope given by $\left|\mathrm{H}\left(\omega_{k}\right)\right|^{2}$. The width of this envelope is the same as that of the background, $\Delta \omega$, which is much larger than the spike width $\delta \omega_{k}$, and hence the spectrum contains about $2 / \eta$ well-separated peaks. The amplitude of the $k$-th peak is proportional to $\mathrm{M}\left(\omega_{k}\right)$.

The noise spectra thus provides one with information useful for the understanding of the microscopic origin of the current quantization. In particular, measuring the Fano factors, allows one to determine the trapping probabilities $p_{n}$. Then for a trapping process which occurs via tunneling, $p_{n}$ are expected to be temperature independent, while temperature-dependent probabilities will indicate phonon-assisted trapping.

The spectral width of the current noise is related to the size of the state, in which the electrons are trapped. Presumably, that length will be longer for two electrons than for a single one, $a_{2}>a_{1}$. This means that near the first threshold the noise spectrum is broader, compared to the second one, $(\Delta \omega)_{1}>(\Delta \omega)_{2}$.

In summary, we have proposed a phenomenological model for the fluctuations of the quantized acoustoelectric current, and derived detailed predictions for the noise spectrum of the current. In particular, we have found that measuring that noise gives additional information which may turn out to be important in understanding the microscopic mechanism of the current quantization.

\section{ACKNOWLEDGMENTS}

This work has been done at the Centre of Advanced Studies, Oslo, Norway, and during the visit of YMG to the Weizmann Institute of Science. Supports from the OEC Project - Access to Submicron Center for Research on Semiconductor Materials, Devices and Structures (HPRI-CT-1999-0026) and the Israel Science Foundation are acknowledged. YMG thanks V. I. Talyanskii for discussions related to the experiment.

[1] A. Wixforth, J. P. Kotthaus, and G. Weimann, Phys. Rev. Lett. 56, 2104 (1986); A. Wixforth, J. Scriba, M. Wassermeir, J. P. Kotthaus, G. Weimann, and W. Schlapp, Phys. Rev. B 40, 7874 (1989); I. L. Drichko, A. M. Diakonov, I. Yu. Smirnov, Y. M. Galperin, and A. I. Toropov, Phys. Rev. B 62, 7470 (2000).

[2] R. L. Willet, M. A. Paalanen, K. W. West, L. N. Pfeiffer, and D. J. Bishop, Phys. Rev. Lett. 65, 112 (1990); R. L. Willet, R. R. Ruel, M. A. Paalanen, K. W. West, and L. N. Pfeiffer, Phys. Rev. B 47, 7344 (1993).

[3] J. M. Shilton, D. R. Mace, V. I. Talyanskii, M. Pepper, M. Y. Simmons, A. C. Churchill, and D. A. Ritchie, Phys. Rev. B 51, 14770 (1995).

[4] J. M. Shilton, D. R. Mace, V. I. Talyanskii, Yu. Galperin, M. Y. Simmons, M. Pepper, and D. A. Ritchie, J. Phys.: Condens. Matter 8, L337 (1996).

[5] J. M. Shilton, V. I. Talyanskii, M. Pepper, D. A. Ritchie, J. E. F. Frost, C. J. Ford, C. G. Smith, and G. A. C. Jones, J. Phys.: Condens. Matter 8, L531 (1996).

[6] V. I. Talyanskii, J. M. Shilton, M. Pepper, C. G. Smith, C. J. Ford, E. H. Linfield, D. A. Ritchie, and G. A. C. Jones, Phys. Rev. B 56, 15180 (1997).

[7] H. Totland and Y. M. Galperin, Phys. Rev. B 54, 8814 (1996); Physica scripta 69, 302 (1997).

[8] V. L. Gurevich, V. B. Pevzner, and G. J. Iafrate, Phys. Rev. Lett. 77, 3881 (1996); V. L. Gurevich, V. I. Kozub, and V. B. Pevzner, Phys. Rev. B 58, 13088 (1998).

[9] D. J. Thouless, Phys. Rev. B 27, 6083 (1983).

[10] G. R. Aizin, G. Gumbs, and M. Pepper, Phys. Rev. B 58, 10589 (1998).

[11] P. A. Maksym, Phys. Rev. B 61, 4727 (2000).

[12] K. Flensberg, Q. Niu, and M. Pustilnik, Phys. Rev. B 60, R16 291 (1999).

[13] S. M. Rytov, Yu. Kravtsov, and V. I. Tatarsky, Principles of Statistical Radiophysics, Springer, Berlin (1989), part 2.

[14] Sh. Kogan, Electronic Noise and Fluctuations in Solids, Cambridge University Press (1996), p. 14.

[15] Our result for $S(\omega)$ is formally not valid for $\omega=0$. The zero frequency is, however, not relevant, since the lowest frequency accessible in the experiment is larger than the inverse measuring (averaging) time $T$. Only for such frequencies the random process can be considered as a stationary one.

[16] G. B. Lesovik, JETP Lett. 49, 592 (1989). 